\title{
Top-down estimate of surface flux in the Los Angeles Basin using a mesoscale inverse modeling technique: Assessing anthropogenic emissions of $\mathrm{CO}$, NOx and $\mathrm{CO} 2$ and their impacts
}

J. Brioude 1,2, W.M. Angevine 1,2, R. Ahmadov ${ }^{1,2}$, S-W Kim ${ }^{1,2}$, S. Evan ${ }^{2}$, S.A. McKeen ${ }^{1,2}$, E.-Y Hsie ${ }^{1,2}$, G.J. Frost ${ }^{1,2}$, J.A. Neuman ${ }^{1,2}$, I.B. Pollack ${ }^{1,2}$, J. Peischl ${ }^{1,2}$, T.B. Ryerson ${ }^{2}$, J. Holloway ${ }^{1,2}$, S.S. Brown ${ }^{2}$, J.B. Nowak ${ }^{1,2}$, J.M. Roberts ${ }^{1,2}$, S.C. Wofsy ${ }^{3}$, G.W. Santoni ${ }^{3}$, T. Oda, ${ }^{4,5}$ M. Trainer $^{2}$

1 Cooperative Institute for Research in Environmental Sciences, University of Colorado, Boulder, Colorado, USA

2 Chemical Sciences Division, Earth System Research Laboratory, National Oceanic and Atmospheric Administration, Boulder, Colorado, USA

${ }^{3}$ Harvard University, School of Engineering and Applied Sciences and Department of Earth and Planetary Sciences, Cambridge, Massachusetts, USA

${ }^{4}$ Cooperative Institute for Research in Atmospheres, Colorado State University, Fort Collins, Colorado, USA

5 Global Monitoring Division, Earth System Research Laboratory, National Oceanic and Atmosphere Administration, Boulder, Colorado, USA

Correspondence to: J. Brioude (Jerome.brioude@noaa.gov)

\section{Supplement material}

Along with NEI 2005 and the posterior estimates, a CARB 2010 projection is also used in the WRF-Chem v3.4 Eulerian model simulations of tropospheric chemistry (See section 4 for details). To downscale the CARB 2010 projection to a 4x4km inventory, the NEI-05 emission 
inventory is modified for 6 counties in Southern California according to the CARB (California Air Resources Board) 2010 projected emissions available at: http://www.arb.ca.gov/app/emsinv/fcemssumcat2009.php. The modified counties are Los Angeles, Orange, Riverside, San Bernardino, Ventura and San Diego. Modifications are based on the CARB grown and controlled projected emissions for a summertime day by modifying emission sums of 20 categories based on the U.S. source classification codes (SCCs) within the NEI. These categories and their corresponding assignments to the CARB classifications are listed in Table 1. The NEI categories chosen for modification are based on the major sources of criteria pollutants (reactive VOC, $\mathrm{NOx}, \mathrm{CO}, \mathrm{SO}_{2}$, and $\mathrm{PM}_{2.5}$ ) for Southern California counties within the 2 inventories. "Point" sources within NEI-05 are not modified in this version. These generally represent a minor contribution to the county totals for most criteria pollutants, and more recent, monitoring based emissions estimates of "point" sources would be the most appropriate choice for inclusion. Only emissions for the "mobile onroad", "mobile nonroad", and "nonpoint" emission sectors within the NEI-05 are modified. Modification factors for the 20 categories within Table 1 were determined by ratioing CARB published emissions to the July monthly averages in the NEI-05 for each county. The total emissions in each inventory that are not in the 20 categories are also determined, and the CARB-10 to NEI05 ratio is used to adjust the "nonpoint" emissions within the uncategorized class. By maintaining the original "point" emissions, the modified NEI-05 inventory is not quantitatively identical to the CARB-10 inventory. However, the disagreement is within $24 \%$ for all species and counties, which is well within the uncertainties of the inventories. Further partitioning within SCC category limits, weekday/weekend and hourly temporal allocations, 4-km grid spatial partitioning, and species allocation for reactive VOC and PM2.5 are kept unchanged within the NEI-05.

Most categories have well defined or obvious category assignments between the 2 inventories. However, a few individual source categories do not have 1-to-1 assignments, and some explanation is necessary:

1) The CARB inventory only reports "natural gas" sources (in their offroad equipment category) whereas the NEI05 splits natural gas sources into "LPG" or "CNG". The CARB natural gas sources are split between the two NEI-05 sources using the NEI-05 relative fractions of LPG and CNG. 
2) The NEI-05 inventory splits airport emissions between "point" emissions and "nonpoint" emissions, and only the "nonpoint" fraction is modified here. For Los Angeles county, the NEI-05 airport emissions are $\sim 85 \%$ of the total, so only $\sim 15 \%$ would undergo modification from ratios of the totals shown in Table 1. Since the NEI-05 and CARB-10 airport emissions are quite similar for Los Angeles county, there are only minor modifications. For the other 5 counties, the "nonpoint" airport sources dominate, and airport emission modifications more directly reflect the CARB-10 inventory.

3) Some very minor sources in the mobile on-road and non-road NEI categories are not modified. This includes railway maintenance equipment and diesel powered recreational boats.

Table 1. Emission categories and Source Classification Code ranges within the NEI-05 inventory that are modified according to CARB 2010 projected emissions. The CARB classifications used to derive the modification factors are in column 3. Summertime emissions of 5 criteria air pollutants, and a designation of their source for Los Angeles county are in the last 6 columns. Units of emissions are in short tons per day.

\begin{tabular}{|c|c|c|c|c|c|c|c|c|}
\hline Onroad Diesel & $\begin{array}{l}2230000000- \\
2239999999\end{array}$ & $\begin{array}{l}\text { light heavy duty diesel trucks-(1\&2) } \\
\text { medium heavy duty diesel trucks } \\
\text { heavy heavy duty diesel trucks } \\
\text { heavy duty diesel urban buses }\end{array}$ & $\begin{array}{l}7.41 \\
8.93\end{array}$ & $\begin{array}{r}98.90 \\
136.57\end{array}$ & $\begin{array}{l}37.11 \\
39.53\end{array}$ & $\begin{array}{l}0.83 \\
0.16\end{array}$ & $\begin{array}{l}2.87 \\
5.06\end{array}$ & $\begin{array}{l}\text { NEI05 } \\
\text { CARB }\end{array}$ \\
\hline Onroad Gasoline & $\begin{array}{l}2200000000- \\
2209999999\end{array}$ & $\begin{array}{l}\text { light duty- passenger, trucks }(1 \& 2) \\
\text { medium duty trucks } \\
\text { gas trucks - light heavy duty ( } 1 \& 2) \text {, } \\
\text { medium and heavy heavy duty. } \\
\text { motorcycles, school buses, other } \\
\text { buses and motor homes }\end{array}$ & $\begin{array}{l}176.33 \\
105.80\end{array}$ & $\begin{array}{l}176.61 \\
106.45\end{array}$ & $\begin{array}{l}1717.2 \\
1039.1\end{array}$ & $\begin{array}{l}1.66 \\
1.15\end{array}$ & $\begin{array}{l}2.73 \\
5.89\end{array}$ & $\begin{array}{l}\text { NEI05 } \\
\text { CARB }\end{array}$ \\
\hline
\end{tabular}




\begin{tabular}{|c|c|c|c|c|c|c|c|c|}
\hline Offroad Gas 2-stroke & $\begin{array}{l}2260000000- \\
2260999999\end{array}$ & $\begin{array}{l}2 \text { stroke - off-road equipment, farm } \\
\text { equipment, recreational vehicles }\end{array}$ & $\begin{array}{l}31.32 \\
15.29\end{array}$ & $\begin{array}{l}0.37 \\
0.28\end{array}$ & $\begin{array}{l}68.24 \\
32.19\end{array}$ & .001 & $\begin{array}{l}1.768 \\
0.088\end{array}$ & $\begin{array}{l}\text { NEI05 } \\
\text { CARB }\end{array}$ \\
\hline Offroad LPG & $\begin{array}{l}2267000000- \\
2267999999\end{array}$ & Natural gas off-road equipment & $\begin{array}{l}6.164 \\
0.132\end{array}$ & $\begin{array}{r}22.664 \\
5.005\end{array}$ & $\begin{array}{l}105.28 \\
32.229\end{array}$ & $\begin{array}{l}.028 \\
0.00\end{array}$ & $\begin{array}{l}0.129 \\
0.070\end{array}$ & $\begin{array}{l}\text { NEI05 } \\
\text { CARB }\end{array}$ \\
\hline $\begin{array}{l}\text { Pleasurecraft 2-stroke } \\
\text { (Offroad) }\end{array}$ & $\begin{array}{l}2282000000- \\
2282009999\end{array}$ & Recreational boats -2 stroke & $\begin{array}{r}6.533 \\
19.928\end{array}$ & $\begin{array}{l}0.202 \\
2.002\end{array}$ & $\begin{array}{l}11.761 \\
31.202\end{array}$ & $\begin{array}{l}.0003 \\
.006\end{array}$ & $\begin{array}{l}0.104 \\
2.030\end{array}$ & $\begin{array}{l}\text { NEI05 } \\
\text { CARB }\end{array}$ \\
\hline $\begin{array}{l}\text { Pleasurecraft 4-stroke } \\
\text { (Offroad) }\end{array}$ & $\begin{array}{l}2282010000- \\
2282019999\end{array}$ & Recreational boats - 4 stroke & $\begin{array}{l}0.797 \\
8.031\end{array}$ & $\begin{array}{l}0.484 \\
4.846\end{array}$ & $\begin{array}{r}9.292 \\
111.47\end{array}$ & $\begin{array}{l}.0003 \\
.008\end{array}$ & $\begin{array}{l}.004 \\
.047\end{array}$ & $\begin{array}{l}\text { NEI05 } \\
\text { CARB }\end{array}$ \\
\hline $\begin{array}{l}\text { Commercial Marine and } \\
\text { Harborcraft Diesel }\end{array}$ & $\begin{array}{l}2280002000- \\
2280002999\end{array}$ & $\begin{array}{l}\text { Ocean going Vessels - Diesel } \\
\text { Commercial Harbor Craft - Diesel }\end{array}$ & $\begin{array}{l}4.017 \\
1.686\end{array}$ & $\begin{array}{r}120.80 \\
34.94\end{array}$ & $\begin{array}{r}21.43 \\
6.15\end{array}$ & $\begin{array}{r}25.98 \\
0.82\end{array}$ & $\begin{array}{l}4.706 \\
0.886\end{array}$ & $\begin{array}{l}\text { NEI05 } \\
\text { CARB }\end{array}$ \\
\hline Railroad & $\begin{array}{l}2285000000- \\
2285999999\end{array}$ & Other Mobile - Trains & $\begin{array}{l}1.202 \\
1.498\end{array}$ & $\begin{array}{l}24.201 \\
11.956\end{array}$ & $\begin{array}{l}3.97 \\
4.51\end{array}$ & $\begin{array}{l}1.230 \\
0.096\end{array}$ & $\begin{array}{l}0.557 \\
0.468\end{array}$ & $\begin{array}{l}\text { NEI05 } \\
\text { CARB }\end{array}$ \\
\hline Architectural Coatings & $\begin{array}{l}2401001000- \\
2401003999\end{array}$ & $\begin{array}{l}\text { Architectural Coating and related } \\
\text { Process solvents }\end{array}$ & $\begin{array}{l}32.695 \\
17.398\end{array}$ & $\begin{array}{l}0.00 \\
0.00\end{array}$ & $\begin{array}{l}0.00 \\
0.00\end{array}$ & $\begin{array}{l}0.00 \\
0.00\end{array}$ & $\begin{array}{l}0.00 \\
0.00\end{array}$ & $\begin{array}{l}\text { NEI05 } \\
\text { CARB }\end{array}$ \\
\hline $\begin{array}{l}\text { Miscellaneous } \\
\text { nonindustrial solvents }\end{array}$ & $\begin{array}{l}2460000000- \\
2465999999\end{array}$ & Consumer products & $\begin{array}{l}70.878 \\
65.208\end{array}$ & $\begin{array}{l}0.00 \\
0.00\end{array}$ & $\begin{array}{l}0.00 \\
0.00\end{array}$ & $\begin{array}{l}0.00 \\
0.00\end{array}$ & $\begin{array}{l}0.00 \\
0.00\end{array}$ & $\begin{array}{l}\text { NEI05 } \\
\text { CARB }\end{array}$ \\
\hline $\begin{array}{l}\text { Petroleum products } \\
\text { storage and transport }\end{array}$ & $\begin{array}{l}2500000000- \\
2509999999\end{array}$ & $\begin{array}{l}\text { Petroleum Marketing } \\
\text { Other mobile-fuel store and handle }\end{array}$ & $\begin{array}{l}42.802 \\
27.872\end{array}$ & $\begin{array}{l}0.00 \\
0.00\end{array}$ & $\begin{array}{l}0.00 \\
0.00\end{array}$ & $\begin{array}{l}0.00 \\
0.00\end{array}$ & $\begin{array}{l}0.00 \\
0.00\end{array}$ & $\begin{array}{l}\text { NEI05 } \\
\text { CARB }\end{array}$ \\
\hline Livestock & $2805000000-$ & Areawide Sources - Farming & 0.536 & 0.00 & 0.00 & 0.00 & 0.020 & NEI05 \\
\hline
\end{tabular}




\begin{tabular}{|c|c|c|c|c|c|c|c|c|}
\hline & 2805059999 & Operations & 0.308 & 0.00 & 0.00 & 0.00 & 0.000 & CARB \\
\hline \multirow[t]{2}{*}{ Commercial cooking } & 2302002000 & Areawide Sources - Cooking & 0.747 & 0.00 & 0.18 & 0.00 & 7.269 & NEI05 \\
\hline & 2302003299 & & 1.223 & 0.00 & 0.00 & 0.00 & 8.776 & CARB \\
\hline Fugitive dust - & $2294000000-$ & Areawide Sources - & 0.00 & 0.00 & 0.00 & 0.00 & 3.018 & NEI05 \\
\hline paved roads & 2294015999 & Paved road dust & 0.00 & 0.00 & 0.00 & 0.00 & 10.96 & CARB \\
\hline Fugitive dust - & $2296000000-$ & Areawide Sources - & 0.00 & 0.00 & 0.00 & 0.00 & 2.880 & NEI05 \\
\hline unpaved roads & 2296005999 & Unpaved road dust & 0.00 & 0.00 & 0.00 & 0.00 & 2.783 & CARB \\
\hline NEI-05 Total & All & All & 580.32 & 605.74 & 3727.6 & 78.73 & 45.23 & NEI05 \\
\hline CARB-10 Total & & All & 367.60 & 506.01 & 1789.7 & 50.01 & 64.83 & CARB \\
\hline Modified NEI-05 Total & All & All & 384.31 & 505.79 & 1777.4 & 60.59 & 61.63 & \\
\hline
\end{tabular}

Note: Aircraft emissions for NEI-05 include the "point" emissions in addition to the "nonpoint" SCC categories listed here. 